\title{
On properties of solutions to Black-Scholes-Barenblatt equations
}

\section{Xinpeng $\mathrm{Li}^{1}$, Yiqing $\mathrm{Lin}^{2}$ and Weicheng $\mathrm{Xu}^{3^{*}}$}

"Correspondence:

xuweicheng@ouc.edu.cn

${ }^{3}$ Department of Finance, Ocean University of China, Qingdao, China

Full list of author information is

available at the end of the article

\begin{abstract}
This paper is concerned with the Black-Scholes-Barenblatt equation $\partial_{t} u+r\left(x \partial_{x} u-u\right)+G\left(x^{2} \partial_{x x} u\right)=0$, where $G(\alpha)=\frac{1}{2}\left(\bar{\sigma}^{2}-\underline{\sigma}^{2}\right)|\alpha|+\frac{1}{2}\left(\bar{\sigma}^{2}+\underline{\sigma}^{2}\right) \alpha, \alpha \in \mathbb{R}$. This equation is usually used for derivative pricing in the financial market with volatility uncertainty. We discuss a strict comparison theorem for Black-Scholes-Barenblatt equations, and study strict sub-additivity of their solutions with respect to terminal conditions.
\end{abstract}

MSC: $35 \mathrm{~B} 05 ; 60 \mathrm{H} 30 ; 91 \mathrm{~B} 28$

Keywords: Black-Scholes-Barenblatt equation; Strict comparison theorem; Strict sub-additivity

\section{Introduction}

The Black-Scholes-Barenblatt (BSB) equation is a generalization of the Black-Scholes (BS) partial differential equation (see Black and Scholes [2]), which is used to describe the extremal of non-arbitrageable prices for derivatives while the underlying asset has volatility uncertainty within a range (see Avellaneda et al. [1]). This type of fully nonlinear parabolic equation is of the following form:

$$
\partial_{t} u+r\left(x \partial_{x} u-u\right)+G\left(x^{2} \partial_{x x} u\right)=0,
$$

where $G$ is a nondecreasing sublinear function defined on $\mathbb{R}$.

Adapting to [1], we first introduce the outline of derivative pricing in the market with volatility uncertainty by using the BSB equation. Precisely, we consider the derivative with maturity date $T$ based on a single liquidly traded stock $S$, which satisfies the Itô equation

$$
d S_{t}=S_{t}\left(r d t+\sigma_{t} d B_{t}\right)
$$

where $B$ is a 1-dimensional Brownian motion and $\sigma$ is a non-anticipative function such that, for strictly positive constants $\underline{\sigma}$ and $\bar{\sigma}$,

$$
0<\underline{\sigma} \leq \sigma_{t} \leq \bar{\sigma}
$$

For simplicity, we always assume that the riskless interest rate $r$ is zero and $S_{0}=1$. The results obtained in this paper can be easily generalized to the case for $r>0$ and any $S_{0}>0$.

(c) The Author(s) 2019. This article is distributed under the terms of the Creative Commons Attribution 4.0 International License (http://creativecommons.org/licenses/by/4.0/), which permits unrestricted use, distribution, and reproduction in any medium, provided you give appropriate credit to the original author(s) and the source, provide a link to the Creative Commons license, and indicate if changes were made. 
We denote by $\mathcal{P}$ the class of all probability measures on the set of paths $\left\{S_{t}\right\}_{0 \leq t \leq T}$ such that (1.2) holds for some $\sigma$ satisfying (1.3). Denote the payoff of the derivative at maturity date by $\varphi\left(S_{T}\right)$. If there is no arbitrage opportunity and the assumption on volatility is correct, then the value of such derivative at time $t \in[0, T]$ should lie between the bounds

$$
u^{\varphi}\left(S_{t}, t\right)=\sup _{P \in \mathcal{P}} \mathbb{E}_{t}^{P}\left[\varphi\left(S_{T}\right)\right]
$$

and

$$
v^{\varphi}\left(S_{t}, t\right)=\inf _{P \in \mathcal{P}} \mathbb{E}_{t}^{P}\left[\varphi\left(S_{T}\right)\right]
$$

which can be regarded as optimal risk-averse ask and bid prices for the derivative at time $t$. As $u^{\varphi}\left(S_{t}, t\right)=-v^{-\varphi}\left(S_{t}, t\right)$, we only discuss the properties of $u^{\varphi}$ in this paper.

In fact, viewing (1.4) and (1.5) as solutions of the stochastic control problems with the control variable $\sigma$, by Krylov [6], we can obtain $u^{\varphi}$ by solving the following BSB equation:

$$
\begin{cases}\partial_{t} u^{\varphi}+G\left(x^{2} \partial_{x x} u^{\varphi}\right)=0, & (x, t) \in \mathbb{R}_{+} \times[0, T) \\ u^{\varphi}(x, T)=\varphi(x), & x \in \mathbb{R}_{+},\end{cases}
$$

where

$$
G(\alpha):=\frac{1}{2}\left(\bar{\sigma}^{2} \alpha^{+}-\underline{\sigma}^{2} \alpha^{-}\right)=\frac{1}{2}\left(\bar{\sigma}^{2}-\underline{\sigma}^{2}\right)|\alpha|+\frac{1}{2}\left(\bar{\sigma}^{2}+\underline{\sigma}^{2}\right) \alpha, \quad \alpha \in \mathbb{R} .
$$

In particular, if $\underline{\sigma}=\bar{\sigma}=\sigma$, (1.6) becomes the well-known Black-Scholes PDE with $r=0$. An equivalent formulation of the above problem is also discussed in the sublinear expectation framework introduced by Peng in [7-9]. In this framework, a new type of Brownian motion with volatility uncertainty in the range of $[\underline{\sigma}, \bar{\sigma}]$ is created. Assume that the dynamics of $S$ is characterized by a diffusion process driven by $G$-Brownian motion $B^{G}$,

$$
d S_{t}=S_{t} d B_{t}^{G}
$$

By the fully nonlinear Feynman-Kac formula (Theorem 4.5 in $\mathrm{Hu}$ et al. [5]), $u^{\varphi}(x, t):=$ $\mathbb{E}^{G}\left[\varphi\left(x \frac{S_{T}}{S_{t}}\right)\right]$ defines the unique solution of BSB equation (1.6), where $\mathbb{E}^{G}$ is the sublinear expectation associated with the G-Brownian motion.

We denote the optimal risk-averse ask price at time 0 defined in (1.4) by a functional $V_{T}: \varphi \mapsto V_{T}(\varphi)$. As explained above, $V_{T}(\varphi)=u^{\varphi}\left(S_{0}, 0\right)=u^{\varphi}(1,0)$. Since the function $G(\cdot)$ defined by (1.7) is a nondecreasing and sublinear function, we can see that $V_{T}(\varphi) \leq V_{T}(\psi)$ if $\varphi \leq \psi$ and $V_{T}(\varphi+\psi) \leq V_{T}(\varphi)+V_{T}(\psi)$. In this paper, we are interested in finding conditions on $\varphi$ and $\psi$ such that the following strict properties hold:

$$
\begin{aligned}
& V_{T}(\varphi)<V_{T}(\psi), \quad \text { if } \varphi \leq \psi ; \\
& V_{T}(\varphi+\psi)<V_{T}(\varphi)+V_{T}(\psi) .
\end{aligned}
$$

These properties are meaningful in the non-arbitrage pricing for derivatives and the portfolio risk-diversification in the market with volatility uncertainty. 
The rest of this paper is organized as follows. In Sect. 2, we give some properties of solutions of the BSB equations. In Sect. 3, we provide a strict comparison theorem for the BSB equations and study strict sub-additivity of their solutions with respect to terminal conditions in Sect. 4.

\section{Preliminaries}

Since PDE (1.6) is the fully nonlinear partial differential equation of second order, the classical smooth solution may not exist. The notion of viscosity solution is that it allows merely continuous functions to be solutions of (1.6), that it provides very general existence and uniqueness theorems.

Definition 2.1 A viscosity subsolution (resp. supsolution) of (1.6) on $(0, T) \times \mathbb{R}$ is an upper (resp. lower) semicontinuous function $u$ such that, for all $(t, x) \in(0, T) \times \mathbb{R}, \phi \in C^{2}((0, T) \times$ $\mathbb{R}$ ) such that $u(t, x)=\phi(t, x)$ and $u<\phi$ (resp. $u>\phi)$ on $(0, T) \times \mathbb{R} \backslash(t, x)$, we have

$$
\partial_{t} u^{\varphi}+G\left(x^{2} \partial_{x x} u^{\varphi}\right) \geq 0 \quad(\text { resp. } \leq 0)
$$

A viscosity solution of (1.6) on $(0, T) \times \mathbb{R}$ is a function that is simultaneously a viscosity subsolution and a viscosity supsolution of $(1.6)$ on $(0, T) \times \mathbb{R}$.

Let $C_{l, \text { Lip }}\left(\mathbb{R}_{+}\right)$be the collection of all locally Lipschitz functions $\varphi$ on $\mathbb{R}_{+}$satisfying

$$
|\varphi(x)-\varphi(y)| \leq C\left(1+|x|^{m}+|y|^{m}\right)|x-y|, \quad \forall x, y \in \mathbb{R}_{+}
$$

where $C>0$ and $m \in \mathbb{N}$ depending on $\varphi$. We have the following solvability result for the viscosity solution of BSB equation (1.6) (see Theorem 7 in Gozzi and Vargiolu [4]).

Theorem 2.2 For each $\varphi \in C_{l, \mathrm{Lip}}\left(\mathbb{R}_{+}\right)$, BSB equation (1.6) has a unique viscosity solution $u^{\varphi}$.

For fixed $T>0$, we define a functional $V_{T}$ by

$$
V_{T}(\varphi)=u^{\varphi}(1,0), \quad \varphi \in C_{l, \mathrm{Lip}}\left(\mathbb{R}_{+}\right)
$$

where $u^{\varphi}$ is the solution of BSB equation (1.6) with terminal condition $u^{\varphi}(x, T)=\varphi(x)$. In fact, $V_{T}(\varphi)$ defines the optimal risk-averse ask price for the European type derivative with payoff function $\varphi\left(S_{T}\right)$ at its maturity.

Since the function $G(\cdot)$ defined by $(1.7)$ is nondecreasing and sublinear on $\mathbb{R}$ with $G(0)=$ 0 , we immediately have the following properties for the functional $V_{T}$.

Proposition 2.3 For $T>0$, the functional $V_{T}(\cdot)$ is a sublinear expectation on $C_{l, \mathrm{Lip}}\left(\mathbb{R}_{+}\right)$, i.e., $\forall \varphi, \psi \in C_{l, \text { Lip }}\left(\mathbb{R}_{+}\right)$, the following properties hold:

(i) Monotonicity: If $\varphi \geq \psi$, then $V_{T}(\varphi) \geq V_{T}(\psi)$;

(ii) Constant preserving: $V_{T}(c)=c$ for all $c \in \mathbb{R}_{+}$;

(iii) Sub-additivity: $V_{T}(\varphi+\psi) \leq V_{T}(\varphi)+V_{T}(\psi)$;

(iv) Positive homogeneity: $V_{T}(\lambda \varphi)=\lambda V_{T}(\varphi)$ for all $\lambda \geq 0$. 
The proposition below compares the BSB equation with the BS equation taking the parameter $\sigma \in[\underline{\sigma}, \bar{\sigma}]$. Let $V_{T}^{\sigma}(\varphi):=u^{\sigma, \varphi}(1,0)$, where $u^{\sigma, \varphi}$ denotes the solution of the BS equation with parameter $\sigma>0$. Recall that if $\varphi(x) \leq \psi(x), \forall x \in \mathbb{R}_{+}$, and if there exists $x_{0} \in \mathbb{R}_{+}$ such that $\varphi\left(x_{0}\right)<\psi\left(x_{0}\right)$, then

$$
V_{T}^{\sigma}(\varphi)<V_{T}^{\sigma}(\psi)
$$

Proposition 2.4 Let $T>0$ and $\varphi \in C_{l, \mathrm{Lip}}\left(\mathbb{R}_{+}\right)$. We have

(i) $V_{T}(\varphi) \geq \sup _{\underline{\sigma} \leq \sigma \leq \bar{\sigma}} V_{T}^{\sigma}(\varphi)$;

(ii) $V_{T}(\varphi)=V_{T}^{\frac{\sigma}{T}}(\varphi)$ if and only if $\varphi$ is concave;

(iii) $V_{T}(\varphi)=V_{T}^{\bar{\sigma}}(\varphi)$ if and only if $\varphi$ is convex;

(iv) Let $\sigma \in(\underline{\sigma}, \bar{\sigma})$, then $V_{T}(\varphi)=V_{T}^{\sigma}(\varphi)$ if and only if $\varphi$ is linear.

Proof Recall that

$$
G(\alpha)=\frac{1}{2} \bar{\sigma}^{2} \alpha^{+}-\frac{1}{2} \sigma^{2} \alpha^{-} \geq \frac{1}{2} \sigma^{2} \alpha
$$

By the comparison theorem for parabolic PDEs (see, e.g., Crandall et al. [3]), we know that $u^{\varphi}(x, t) \geq u^{\sigma, \varphi}(x, t), \forall(x, t) \in \mathbb{R}_{+} \times(0, T)$, then (i) follows by the continuity of $u^{\varphi}$ and $u^{\sigma, \varphi}$. In order to show (ii) to (iv), we first prove that

$$
V_{T}(\varphi)=V_{T}^{\sigma}(\varphi) \quad \Longleftrightarrow \quad u^{\varphi}(x, t)=u^{\sigma, \varphi}(x, t), \quad \forall(x, t) \in \mathbb{R}_{+} \times(0, T)
$$

The necessity is obvious due to the continuity of $u^{\varphi}$ and $u^{\sigma, \varphi}$. For the sufficiency, we already know from the comparison theorem that $u^{\varphi}(x, t) \geq u^{\sigma, \varphi}(x, t)$, then we suppose that there exists $\left(x_{0}, t_{0}\right) \in \mathbb{R}_{+} \times(0, T)$ such that $u^{\varphi}\left(x_{0}, t_{0}\right)>u^{\sigma, \varphi}\left(x_{0}, t_{0}\right)$. By the strict comparison result (2.1), we have

$$
\begin{aligned}
V_{T}(\varphi) & =V_{t_{0}}\left(u^{\varphi}\left(\cdot, t_{0}\right)\right) \geq V_{t_{0}}^{\sigma}\left(u^{\varphi}\left(\cdot, t_{0}\right)\right) \\
& >V_{t_{0}}^{\sigma}\left(u^{\sigma, \varphi}\left(\cdot, t_{0}\right)\right)=V_{T}^{\sigma}(\varphi)
\end{aligned}
$$

which is a contradiction.

The right-hand side of (2.2) implies that if $V_{T}(\varphi)=V_{T}^{\sigma}(\varphi)$, then the dynamic of BSB equation (1.6) and of the BS equation with the parameter $\sigma$ coincides, i.e.,

$$
G\left(x^{2} \partial_{x x} u^{\sigma, \varphi}(x, t)\right)=\frac{\sigma^{2} x^{2}}{2} \partial_{x x} u^{\sigma, \varphi}(x, t), \quad \forall(x, t) \in \mathbb{R}_{+} \times(0, T) .
$$

To show (ii), first due to the preservation of convexity, we know that $\varphi$ is concave if and only if $\partial_{x x} u^{\sigma, \varphi}(x, t) \leq 0, \forall(x, t) \in \mathbb{R}_{+} \times(0, T)$. This is to say: when $\sigma=\underline{\sigma},(2.3)$ holds for all $(x, t) \in \mathbb{R}_{+} \times(0, T)$, which is equivalent to $V_{T}(\varphi)=V_{T}^{\frac{\sigma}{T}}(\varphi)$ by (2.2). The convex case (iii) can be proved similarly.

Now we turn to verify (iv). When $\sigma \in(\underline{\sigma}, \bar{\sigma})$, it is easy to find that (2.3) holds if and only if $\partial_{x x} u^{\sigma, \varphi}(x, t)=0, \forall(x, t) \in \mathbb{R}_{+} \times(0, T)$, which is equivalent to that $\varphi$ is linear. 


\section{Strict comparison theorem for BSB equations}

In this section, we shall look for conditions such that the strict comparison (1.8) holds. Recall that $0<\underline{\sigma}<\bar{\sigma}$ in this paper.

Theorem 3.1 Fix $T>0$. Let $\varphi, \psi \in C_{l, \text { Lip }}\left(\mathbb{R}_{+}\right)$such that $\varphi(x) \leq \psi(x)$ for all $x \in \mathbb{R}_{+}$. Then $V_{T}(\varphi)<V_{T}(\psi)$ if and only if there exists $x_{0} \in \mathbb{R}_{+}$such that $\varphi\left(x_{0}\right)<\psi\left(x_{0}\right)$.

By Proposition 2.3(iii), we only need to prove that if $\varphi(x) \leq 0$ and there exists $x_{0} \in \mathbb{R}_{+}$ such that $\varphi\left(x_{0}\right)<0$, then $V_{T}(\varphi)<0$. In order to prove this property, we first change variables by taking $y=\ln x$, then (1.6) becomes the following equation:

$$
\begin{cases}\partial_{t} \tilde{u}+G\left(\partial_{y y} \tilde{u}-\partial_{y} \tilde{u}\right)=0, & (y, t) \in \mathbb{R} \times[0, T) \\ \tilde{u}(y, T)=\tilde{\varphi}(y), & y \in \mathbb{R},\end{cases}
$$

where $\tilde{u}(y, t)=u^{\varphi}(x, t)$ and $\tilde{\varphi}(y)=\varphi(x)$.

Proposition 3.2 Let $\varphi \in C_{l, \mathrm{Lip}}(\mathbb{R})$ such that $\varphi \leq 0$ and there exists $x_{0}$ such that $\varphi\left(x_{0}\right)<0$. Let $\tilde{u}$ be the solution of PDE (3.1) with terminal condition $\tilde{u}(x, T)=\varphi(x)$. Then $\tilde{u}(x, t)<0$ for all $(x, t) \in \mathbb{R} \times[0, T)$.

To prove Proposition 3.2, we need the following lemma.

Lemma 3.3 Fix $a \in \mathbb{R}$. Let $\tilde{u}_{m}$ be the solution of PDE (3.1) with the terminal condition $\tilde{u}_{m}(T, x)=-\exp \left(-\frac{m \theta|x-a|^{2}}{2}\right)$, where $\theta=T+\frac{1}{\sigma^{2}}+1$ and $m \geq 1$. Then, for any $(x, t) \in \mathbb{R} \times[0, T)$, we have

$$
\tilde{u}_{m}(x, t) \leq-(1+m(T-t))^{-\bar{\sigma}^{2} \theta} \exp \left(-\frac{m \theta|x-a|^{2}}{2(1+m(T-t))}\right) .
$$

Proof We denote by $\bar{u}_{m}$ the function on the right-hand side of inequality (3.2). By the comparison theorem, it suffices to show that $\bar{u}_{m}$ is the supersolution of PDE (3.1). Indeed, it is easy to verify that

$$
\begin{aligned}
& \partial_{t} \bar{u}_{m}=\frac{\bar{\sigma}^{2} \theta m}{1+m(T-t)} \bar{u}_{m}-\frac{m^{2} \theta|x-a|^{2}}{2(1+m(T-t))^{2}} \bar{u}_{m}, \\
& \partial_{x} \bar{u}_{m}=-\frac{m \theta(x-a)}{1+m(T-t)} \bar{u}_{m}, \\
& \partial_{x x} \bar{u}_{m}=-\frac{m \theta}{1+m(T-t)} \bar{u}_{m}+\frac{m^{2} \theta^{2}|x-a|^{2}}{(1+m(T-t))^{2}} \bar{u}_{m} .
\end{aligned}
$$

Then, for each $(t, x) \in[0, T) \times \mathbb{R}$, it is derived from $\bar{u}_{m} \leq 0$ that

$$
\begin{aligned}
\partial_{t} \bar{u}_{m} & +G\left(\partial_{x x}^{2} \bar{u}_{m}-\partial_{x} \bar{u}_{m}\right) \\
= & \frac{\bar{\sigma}^{2} \theta m}{1+m(T-t)} \bar{u}_{m}-\frac{m^{2} \theta|x-a|^{2}}{2(1+m(T-t))^{2}} \bar{u}_{m} \\
& \quad-\bar{u}_{m} G\left(\frac{m \theta}{1+m(T-t)}-\frac{m^{2} \theta^{2}|x-a|^{2}}{(1+m(T-t))^{2}}-\frac{m \theta(x-a)}{1+m(T-t)}\right)
\end{aligned}
$$




$$
\begin{aligned}
\leq & \frac{\bar{\sigma}^{2} \theta m}{1+m(T-t)} \bar{u}_{m}-\frac{m^{2} \theta|x-a|^{2}}{2(1+m(T-t))^{2}} \bar{u}_{m} \\
& -\bar{u}_{m} G\left(\frac{m \theta}{1+m(T-t)}-\frac{m^{2} \theta^{2}|x-a|^{2}}{(1+m(T-t))^{2}}+\frac{m \theta\left(|x-a|^{2}+1\right)}{1+m(T-t)}\right) \\
\leq & \frac{\bar{\sigma}^{2} \theta m}{1+m(T-t)} \bar{u}_{m}-\frac{m^{2} \theta|x-a|^{2}}{2(1+m(T-t))^{2}} \bar{u}_{m} \\
& -\bar{u}_{m} G\left(\frac{2 m \theta}{1+m(T-t)}\right)-\bar{u}_{m} G\left(-\frac{m \theta(m(\theta-T+t)-1)|x-a|^{2}}{(1+m(T-t))^{2}}\right) \\
= & \frac{m \theta \bar{u}_{m}(x-a)^{2}}{2(1+m(T-t))^{2}}\left(\underline{\sigma}^{2}(m(\theta-T+t)-1)-m\right) \leq 0 .
\end{aligned}
$$

This completes the proof.

Now we give the proof of Proposition 3.2, from which we can easily see that Theorem 3.1 holds.

Proof of Proposition 3.2 For $0<\varepsilon<\left|\varphi\left(x_{0}\right)\right|$, there exists $\delta>0$ such that $\varphi(x) \leq-\varepsilon$ if $\left|x-x_{0}\right|<\delta$. Then we have, for each $m \geq 1$,

$$
\varphi(x) \leq-\varepsilon e^{-\frac{m \theta\left(x-x_{0}\right)^{2}}{2}}+\varepsilon e^{-\frac{m \theta \delta^{2}}{2}}
$$

where $\theta=T+\frac{1}{\underline{\sigma}^{2}}+1$ as in Lemma 3.3.

By the comparison theorem for PDE (3.1) and Lemma 3.3, we have

$$
\begin{aligned}
\tilde{u}(x, t) & \leq-\varepsilon(1+m(T-t))^{-\bar{\sigma}^{2} \theta} e^{-\frac{m \theta\left(x-x_{0}\right)^{2}}{2(1+m(T-t))}}+\varepsilon e^{-\frac{m \theta \delta^{2}}{2}} \\
& \leq-\varepsilon(1+m(T-t))^{-\bar{\sigma}^{2} \theta} e^{-\frac{\theta\left(x-x_{0}\right)^{2}}{2(T-t)}}+\varepsilon e^{-\frac{m \theta \delta^{2}}{2}}
\end{aligned}
$$

For fixed $(x, t) \in \mathbb{R} \times[0, T)$, we can take $m$ large enough such that

$$
(1+m(T-t))^{-\bar{\sigma}^{2} \theta} e^{-\frac{\theta\left(x-x_{0}\right)^{2}}{2(T-t)}}>e^{-\frac{m \theta \delta^{2}}{2}}
$$

which implies that $\tilde{u}(x, t)<0$.

Corollary 3.4 Fix $T>0$. Let $\varphi, \psi \in C_{l, \mathrm{Lip}}\left(\mathbb{R}_{+}\right)$such that $\varphi(x) \leq \psi(x)$ for all $x \in \mathbb{R}_{+}$. Then $V_{T}(\varphi)=V_{T}(\psi)$ if and only if $u^{\varphi}(x, t)=u^{\psi}(x, t), \forall(x, t) \in \mathbb{R}_{+} \times(0, T)$.

Remark 3.5 The strict comparison does not hold if $\underline{\sigma}=0$. For example, let $\varphi=\min (x, 1)$ and $\psi \equiv 1$. However, when $\underline{\sigma}=0, V_{T}(\varphi)=u^{\varphi}(1,0)=1=u^{\psi}(1,0)=V_{T}(\psi)$.

Remark 3.6 The price $V_{T}(\varphi)$ obtained from BSB equation (1.6) is indeed the least possible initial cost to risklessly hedge a short position in the derivative security $\varphi$ by self-financing portfolios. The properties above show that this type of pricing under volatility uncertainty is arbitrage-free in the sense: if $\psi$ is "substantially" superior to $\varphi$, i.e., $\varphi \leq \psi$ and there exists $x_{0} \in \mathbb{R}_{+}$such that $\varphi\left(x_{0}\right)<\psi\left(x_{0}\right)$, then one could not hedge a short position of $\psi$ with the initial value $V_{T}(\varphi)$. In other words, if two options $\varphi$ and $\psi$ satisfy $\varphi \leq \psi$ but they have 
the same optimal risk-averse ask price given by (1.6), then they generate the same path of their "present values", i.e., $u^{\varphi}\left(S_{t}, t\right)=u^{\psi}\left(S_{t}, t\right), \forall t \in(0, T)$.

\section{Strict sub-additivity for BSB equations}

In the remainder of this paper, we consider the strict sub-additivity (1.9) of the functional $V_{T}$.

Theorem 4.1 Fix $T>0$. For $\varphi, \psi \in C_{l, \text { Lip }}\left(\mathbb{R}_{+}\right)$, we have

$$
V_{T}(\varphi+\psi)<V_{T}(\varphi)+V_{T}(\psi)
$$

if and only if there exists $\left(x_{0}, t_{0}\right) \in \mathbb{R}_{+} \times(0, T)$ such that

$$
u^{\varphi+\psi}\left(x_{0}, t_{0}\right)<u^{\varphi}\left(x_{0}, t_{0}\right)+u^{\psi}\left(x_{0}, t_{0}\right) .
$$

Proof If there exists $\left(x_{0}, t_{0}\right) \in \mathbb{R}_{+} \times(0, T)$ such that (4.2) holds, then by Theorem 3.1 we have

$$
\begin{aligned}
V_{T}(\varphi+\psi) & =V_{t_{0}}\left(u^{\varphi+\psi}\left(\cdot, t_{0}\right)\right)<V_{t_{0}}\left(u^{\varphi}\left(\cdot, t_{0}\right)+u^{\psi}\left(\cdot, t_{0}\right)\right) \\
& \leq V_{t_{0}}\left(u^{\varphi}\left(\cdot, t_{0}\right)\right)+V_{t_{0}}\left(u^{\psi}\left(\cdot, t_{0}\right)\right)=V_{T}(\varphi)+V_{T}(\psi) .
\end{aligned}
$$

If (4.1) holds, which is equivalent to $u^{\varphi+\psi}(1,0)<u^{\varphi}(1,0)+u^{\psi}(1,0)$, then by the continuity of $u^{\varphi+\psi}, u^{\varphi}$, and $u^{\psi}$, there exists $\left(x_{0}, t_{0}\right) \in \mathbb{R}_{+} \times(0, T)$ such that (4.2) holds.

Remark 4.2 The strict inequality (4.1) could be explained as the diversification of volatility risk in the portfolio of European type derivatives (cf. [1]).

The theorem above shows that the optimal risk-averse ask price of the portfolio " $\varphi+\psi$ " will be strictly lower than the sum of the individual prices for " $\varphi$ " and " $\psi$ " if the paths of "present values" $u^{\varphi+\psi}\left(S_{t}, t\right), u^{\varphi}\left(S_{t}, t\right)$, and $u^{\psi}\left(S_{t}, t\right)$ are strictly sub-additive at some time $t_{0} \in(0, T)$. In practice, (4.2) is very difficult to check, thus we look for sufficient but simpler conditions for the strict sub-additivity of $V_{T}$ in what follows.

Theorem 4.3 Fix $T>0$. For $\varphi, \psi \in C_{l, \text { Lip }}\left(\mathbb{R}_{+}\right)$, if there exists $x_{0} \in \mathbb{R}_{+}$such that

$$
\varphi^{\prime \prime}\left(x_{0}\right) \psi^{\prime \prime}\left(x_{0}\right)<0 \text {, }
$$

then we have

$$
V_{T}(\varphi+\psi)<V_{T}(\varphi)+V_{T}(\psi) .
$$

Proof Without loss of generality we can assume that there exists $\varepsilon>0$ such that $\varphi^{\prime \prime}\left(x_{0}\right)>\varepsilon$ and $\psi^{\prime \prime}\left(x_{0}\right)<-\varepsilon$.

Since $\varphi$ is twice differentiable at $x_{0}$, we have

$$
\begin{aligned}
G\left(x_{0}^{2} \varphi^{\prime \prime}\left(x_{0}\right)\right) & =\lim _{\delta \downarrow 0} \frac{u^{\varphi}\left(x_{0}, T-\delta\right)-u^{\varphi}\left(x_{0}, T\right)}{\delta} \\
& =\lim _{\delta \downarrow 0} \frac{u^{\varphi}\left(x_{0}, T-\delta\right)-\varphi\left(x_{0}\right)}{\delta} .
\end{aligned}
$$


Then, by the definition of the function $G$, we can deduce that

$$
\begin{aligned}
\lim _{\delta \downarrow 0} & \frac{u^{\varphi}\left(x_{0}, T-\delta\right)+u^{\psi}\left(x_{0}, T-\delta\right)-u^{\varphi+\psi}\left(x_{0}, T-\delta\right)}{\delta} \\
\quad & G\left(x_{0}^{2} \varphi^{\prime \prime}\left(x_{0}\right)\right)+G\left(x_{0}^{2} \psi^{\prime \prime}\left(x_{0}\right)\right)-G\left(x_{0}^{2}\left(\varphi^{\prime \prime}\left(x_{0}\right)+\psi^{\prime \prime}\left(x_{0}\right)\right)\right) \\
& \geq \frac{\left(\bar{\sigma}^{2}-\underline{\sigma^{2}}\right) x_{0}^{2} \varepsilon}{2} .
\end{aligned}
$$

Thus, there exists $t_{0} \in(0, T)$ such that

$$
u^{\varphi}\left(x_{0}, t_{0}\right)+u^{\psi}\left(x_{0}, t_{0}\right)-u^{\varphi+\psi}\left(x_{0}, t_{0}\right) \geq \frac{\left(\bar{\sigma}^{2}-\underline{\sigma}^{2}\right) x_{0}^{2} \varepsilon}{4}>0 .
$$

Finally, by Theorem 4.1, we have $V_{T}(\varphi+\psi)<V_{T}(\varphi)+V_{T}(\psi)$.

The following corollary is straightforward.

Corollary 4.4 Fix $T>0$. For $\varphi, \psi \in C_{l, \mathrm{Lip}}^{2}\left(\mathbb{R}_{+}\right)$, if $V_{T}(\varphi+\psi)=V_{T}(\varphi)+V_{T}(\psi)$, then we have

$$
\varphi^{\prime \prime}(x) \psi^{\prime \prime}(x) \geq 0, \quad \forall x \in \mathbb{R}_{+} .
$$

Remark 4.5 Unfortunately, the inverse statement of Corollary 4.4 is not true, which means that (4.3) is merely a sufficient condition. We have the following counterexample: Let $\varphi(x)=(\max \{x-2,0\})^{2}$ which is convex and $\psi(x)=-(\min \{x-1,0\})^{2}$ which is concave. It is easy to find that $V_{T}(\varphi+\psi)<V_{T}(\varphi)+V_{T}(\psi)$, but $\varphi^{\prime \prime}(x) \psi^{\prime \prime}(x)=0$ for all $x \in \mathbb{R}_{+}$.

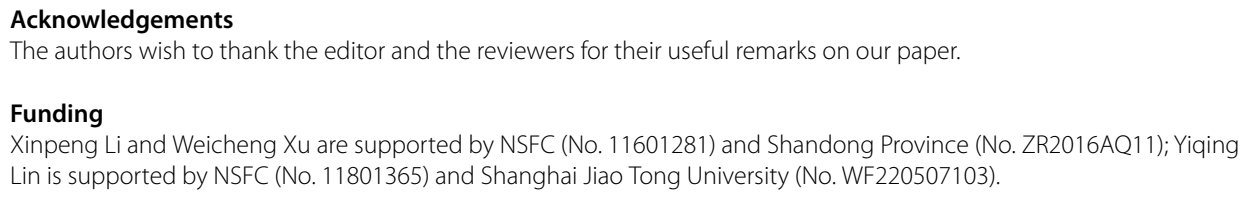

${ }^{1}$ Research Center for Mathematics and Interdisciplinary Sciences and School of Mathematics, Shandong University, Jinan, China. ${ }^{2}$ School of Mathematical Sciences, Shanghai Jiao Tong University, Shanghai, China. ${ }^{3}$ Department of Finance, Ocean University of China, Qingdao, China.

\section{Publisher's Note}

Springer Nature remains neutral with regard to jurisdictional claims in published maps and institutional affiliations.

Received: 10 December 2018 Accepted: 9 May 2019 Published online: 17 May 2019

\section{References}

1. Avellaneda, M., Levy, A., Paras, A.: Pricing and hedging derivative securities in markets with uncertain volatilities. Appl. Math. Finance 2, 73-88 (1995)

2. Black, F., Scholes, M.: The pricing of options and corporate liabilities. J. Polit. Econ. 81, 637-659 (1973)

3. Crandall, G.M., Ishii, H., Lions, P.-L.: User's guide to viscosity solutions of second order partial differential equations. Bull. Am. Math. Soc. 27(1), 1-67 (1992)

4. Gozzi, F., Vargiolu, T.: Superreplication of European multiasset derivatives with bounded stochastic volatility. Math Methods Oper. Res. 55(1), 69-91 (2002) 
5. Hu, M., Ji, S., Peng, S., Song, Y.: Comparison theorem, Feynman-Kac formula and Girsanov transformation for BSDEs driven by G-Brownian motion. Stoch. Process. Appl. 124, 1170-1195 (2014)

6. Krylov, N.V.: Controlled Diffusion Processes. Springer, New York (1980)

7. Peng, S.: G-Expectation, G-Brownian motion and related stochastic calculus of Itô's type. In: Stoch. Anal. Appl. The Abel Symposium, vol. 2, pp. 541-567. Springer, Berlin (2007)

8. Peng, S.: Multi-dimensional G-Brownian motion and related stochastic calculus under G-expectation. Stoch. Process. Appl. 118(12), 2223-2253 (2008)

9. Peng, S.: Nonlinear expectations and stochastic calculus under uncertainty (2010). arXiv:1002.4546v1

Submit your manuscript to a SpringerOpen ${ }^{\circ}$ journal and benefit from:

- Convenient online submission

- Rigorous peer review

- Open access: articles freely available online

- High visibility within the field

- Retaining the copyright to your article

Submit your next manuscript at $\gg$ springeropen.com 